\title{
BMJ Open Quality Implementing screening for social determinants of health using the Core 5 screening tool
}

\author{
Alison Bradywood, ${ }^{1}$ Treasa "Susie" Leming-Lee, ${ }^{2}$ Richard Watters, ${ }^{2}$ \\ Craig Blackmore ${ }^{3}$
}

To cite: Bradywood A, LemingLee T"Susie", Watters R, et al. Implementing screening for social determinants of health using the Core 5 screening tool. BMJ Open Quality 2021;10:e001362. doi:10.1136/ bmjoq-2021-001362

- Additional supplemental material is published online only. To view, please visit the journal online (http://dx.doi.org/10. 1136/bmjoq-2021-001362).

Received 26 January 2021 Accepted 18 July 2021

\section{A) Check for updates}

(C) Author(s) (or their employer(s)) 2021. Re-use permitted under CC BY-NC. No commercial re-use. See rights and permissions. Published by BMJ.

${ }^{1}$ Nursing Administration, Virginia Mason Medical Center, Seattle, Washington, USA

${ }^{2}$ School of Nursing, Vanderbilt University, Nashville, Tennessee, USA

${ }^{3}$ Center for Health Care Improvement Science, Virginia Mason Medical Center, Seattle, Washington, DC, USA

Correspondence to Dr Alison Bradywood; alison.bradywood@ virginiamason.org

\section{ABSTRACT}

Social determinants of health (SDOH) have been documented to underpin $80 \%$ of overall health and are being increasingly recognised as key factors in addressing tertiary health outcomes. Yet, despite the widespread acceptance of the association of SDOH with health outcomes, more than two-thirds of hospitals do not screen for social risk factors that indicate individuallevel adverse SDOH. Such screening for social risk factors represents the first step in connecting patients with resources and documents the prevalence of social needs. The aim of this project was to implement the Core 5 social risk screening tool and evaluate its efficacy and usability in identifying social risk factors in a presurgical spine population. Prior to this implementation, screening for social risk had not been performed. The Model for Improvement provided a framework for implementing and evaluating the Core 5 social risk screening tool. Methods included implementation of a patient self-report social risk screening tool, referral workflow to connect patients with needed resources and evaluation of staff feasibility in using the Core 5 tool. The results indicated that the screening tool identified patients with social risk factors and staff reported perceptions of efficacy and usability in clinical workflow. Overall, 52 of $88(59 \%)$ of subjects in the presurgical spine population were effectively screened. Of these, five patients $(10 \%)$ had identified social needs that needed to be addressed prior to surgery. The staff usability survey for the Core 5 tool demonstrated high acceptance and usability, with an average score of 4.4 (out of 5). Future work should evaluate the efficacy of the screening tool in other ambulatory and tertiary settings.

\section{PROBLEM}

Despite widespread acceptance of the role of social determinants of health $(\mathrm{SDOH})$ in determining health outcomes, screening for individual-level social risk factors in clinical care settings remains minimal. Ohanian and McConnell assert that more than two-thirds of hospitals still do not screen for social risk factors. ${ }^{1-3}$ Until recently, across the Virginia Mason Medical Center (VMMC), SDOH had not been integrated into formal assessments or documentation to consistently capture social risk factors. Lack of routine patient screening resulted in insufficient and inconsistent information leading to late social service referrals (indicated by identification of these issues on the day of discharge), potentially affecting surgical outcomes and extending length of stay (LOS).

Within the surgical spine population at VMMC, SDOH had become a significant concern as LOS for this group demonstrated excess days above benchmark at 5.94 days compared with the 2019 Centers for Medicaid and Medicare (CMS) benchmark of 4.01 days. ${ }^{4}$ Readmission rates for this population were at 4.76/1000 patient days, in comparison to the benchmark range for similar procedures of 4.2 to $7.6 / 1000 .^{5}$ These rates persisted in spite of strict clinical appropriateness guidelines applied to the degenerative spine population since 2014. These guidelines were established by the Washington state-wide Bree Collaborative, a state organisation tasked with improving healthcare quality and cost-effectiveness to optimise patient outcomes. As part of this surgical warranty programme, patients must demonstrate failed physical therapy, glycaemic control, no tobacco use, moderate body mass index and no life-limiting conditions in order to proceed to surgery. ${ }^{6}$ However, in the existing VMMC model, social factors remained largely unaddressed and were anecdotally related to extended hospital LOS. Identification of social risk factors that can influence up to $80 \%$ of health may further improve postsurgical outcomes. ${ }^{1-3}$

The purpose of this quality improvement project was to implement the Core 5 social risk screening tool within a presurgical spine population in order to improve identification of social needs and subsequent referrals to community resources. Long-term outcomes would potentially reduce hospital LOS. The aim was to increase patient selfreport using this tool from $0 \%$ to $90 \%$ in the neurosurgical clinic over a 6 -week period. 
The goal of $90 \%$ was established by VMMC leadership as a target.

\section{BACKGROUND}

SDOH are estimated to underpin $80 \%$ of overall health and well-being and increasingly are recognised as key factors in tertiary health outcomes. ${ }^{7}$ The WHO defines $\mathrm{SDOH}$ as 'the wider set of forces and systems shaping the conditions of daily life'. 7 These structural and social factors include poverty, housing instability, transportation options, language/literacy abilities, employment status, race/ethnicity, education level and social support. $\mathrm{SDOH}$ influence individual 'health-related behaviours, health literacy levels, sense of self-efficacy and access to societal resources including healthcare'. ${ }^{89}$ The impacts of these factors weigh heavily on individual health outcomes and often are cumulatively responsible, along with chronic conditions, for disparities in disabilities, increased hospitalisations and more frequent hospital readmissions. ${ }^{10-13}$

In comparison with other high-income and middleincome countries, the USA consistently scores poorly in overall health outcomes and access to healthcareassociated with socioeconomic factors. ${ }^{14}$ In Washington state, the effects of health disparities have been linked with a 10-year difference in life expectancy between urban and rural counties and a 12-year gap in longevity for particular racial and ethnic groups. ${ }^{5}$ Correspondingly, individuals in Washington state's rural communities and racial/ethnic groups of colour disproportionately experience poverty, attainment only of a high school education and significant housing challenges. ${ }^{5}$ These and other social inequities contribute to difficulties managing existing health conditions and the capacity of communities to support health-promoting actions that mitigate disease incidence. Likewise, such circumstances lead to greater utilisation of high-cost healthcare resources and longer hospitalisations. ${ }^{15}$ To address these outcomes, interventions on $\mathrm{SDOH}$ are needed with a first step of implementing screening for social risk factors. Process improvement metrics such as screening rates, staff acceptance of screening practices and prevalence of social risk factors contribute valuable data in building a business case for improving health equity and reducing high-cost healthcare expenditures.

\section{DESIGN}

This quality improvement project focused on examining the feasibility of implementing a social risk screening tool. Using the Plan-Do-Study-Act (PDSA) process, feasibility was defined in terms of limited impact on staff workload, effective capture of SDOH information, and the ability to prescribe interventions when indicated by patient need. Key steps in this process were to identify a simple, reliable screening tool, pilot a screening process, and assess staff feedback.
Social risk screening tool selection: Core 5 social risk screening tool

Although there are many screening tools published to assess social determinant risk factors, the number of social determinant domains involved, the targeted care setting for screening and the length of tools vary considerably. Another dimension is whether the tools have reliabile data regarding patient responses. Numerous tools were considered against these criteria with overall length and type of care setting being significant considerations. The most common of these are included for review. PRAPARE focuses on community health settings and Medicaid managed care organisations with 16 core measures related to demographics and social risk. ${ }^{6}$ Similarly, the CMS Health-Related Social Needs Screening Tool provides a 10 or 26-question expanded tool focusing on needs of Medicare/Medicaid beneficiaries. ${ }^{16}$ Finally, the 12-question THRIVE tool targets community practitioners, versus a subspecialty or tertiary healthcare setting. ${ }^{17}$ While there is recognition of the value of these tools in identifying social risk factors, the nature of the questionnaires did not adequately capture the need of the care delivery setting and the length was determined to be a potential barrier to implementation.

We selected the Core 5 social risk screening tool as a short tool specific to screening of social risk factors that would be suitable in a subspecialty or tertiary care setting. This tool, developed by the Columbus Ohio Public Health Department, consists of five questions (online supplemental appendix A written at a fifth-grade reading level in patient-friendly language to promote disclosure of sensitive social content. ${ }^{18}$ Pilot studies found consensus in the usability by nursing staff, reported increases in social support referrals for patients, and documented reliability in measurement. ${ }^{18}$

The implementation of the Core 5 social risk tool implementation was preceded by two interventions for the nursing staff, physician assistants, social workers and medical assistant staff involved in this project: (1) background education on research on $\mathrm{SDOH}$ and (2) workflow training on the Core 5 tool, screening process and how to answer patient questions.

\section{Patient population}

The project setting was the neurosurgical specialty clinic associated with an urban, 336-bed tertiary-care hospital, located in downtown Seattle, Washington. The neurosurgical clinic included four neurosurgeons, two orthopaedic spine surgeons, nine physician assistants, two nurses and support staff. Potential participants included all back pain patients seen in the neurosurgical clinic for a 6 -week period of time who progressed to surgery over a 3-month timeframe. All patients received the screening tool in the neurosurgery clinic during their preoperative consult. Patients were subsequently excluded if they: (1) declined surgical intervention at this time, (2) the scheduled surgery was outside of the programme window or (3) according to the surgeon, did not presently meet the Bree Collaborative criteria for surgical intervention. 


\section{STRATEGY}

\section{Model for Improvement}

This project used the Model for Improvement framework to guide this quality improvement study. The model is designed to improve patient care delivery systems and includes three questions: what are we trying to accomplish (the aim); (2) how will we know that a change is an improvement (measurement) and (3) what change can we make that will result in improvement? (the change), along with the PDSA cycle. ${ }^{19}$ The PDSA is an iterative, four-phase problem-solving model used for improving a process or carrying out change. ${ }^{19}$ For this project, the PDSA process was used to plan and rapidly test the utility of the Core 5 social risk screening tool for improving services for $\mathrm{SDOH}$.

\section{PDSA cycle: Plan phase}

During the Plan phase of this project, objectives were developed and used to drive the pilot to determine the feasibility of the Core 5 social risk screening tool and impact on social service referrals. The pilot team was identified based on concerns for a social need within this patient population and the social risk screening tool was selected based on available evidence. Staff workflow was designed by the project lead in collaboration with the department nursing director and staff. Audit tools for staff feasibility were chosen through discussions with the Ohio Action Coalition. The action plan to implement the PDSA cycle included conducting a brief staff education regarding the concept of $\mathrm{SDOH}$ and basic scripting to facilitate patient questions that may arise in completing the screening tool.

\section{PDSA cycle: Do phase}

The Do phase was divided into two steps. The first step involved implementing an educational session by the project PI to staff in the spine clinic. The initial education session focused on educating the nursing staff, physician assistants, social workers, and scheduling staff about the Core 5 social risk screening tool. Additional education sessions focused on $\mathrm{SDOH}$, literature review findings, recommendations regarding how to answer patient questions related to the Core 5 social risk screening tool, and how to implement the Core 5 social risk screening tool.

The second step of the Do phase was the implementation of the Core 5 social risk screening tool to screen all back pain patients seen in the neurosurgical clinic (figure 1). A paper form of the Core 5 social risk screening tool questionnaire is provided to the patients by the clinic scheduler when patients register for their appointment at the front desk of the clinic. This questionnaire accompanied other paper questionnaires related to back pain and function that represent normative procedures for this patient population. All potential participants received a brief description of the rationale for completing social risk factor questions and how the information will be used in comprehensively planning for their care. Staff was trained to this standard scripting as

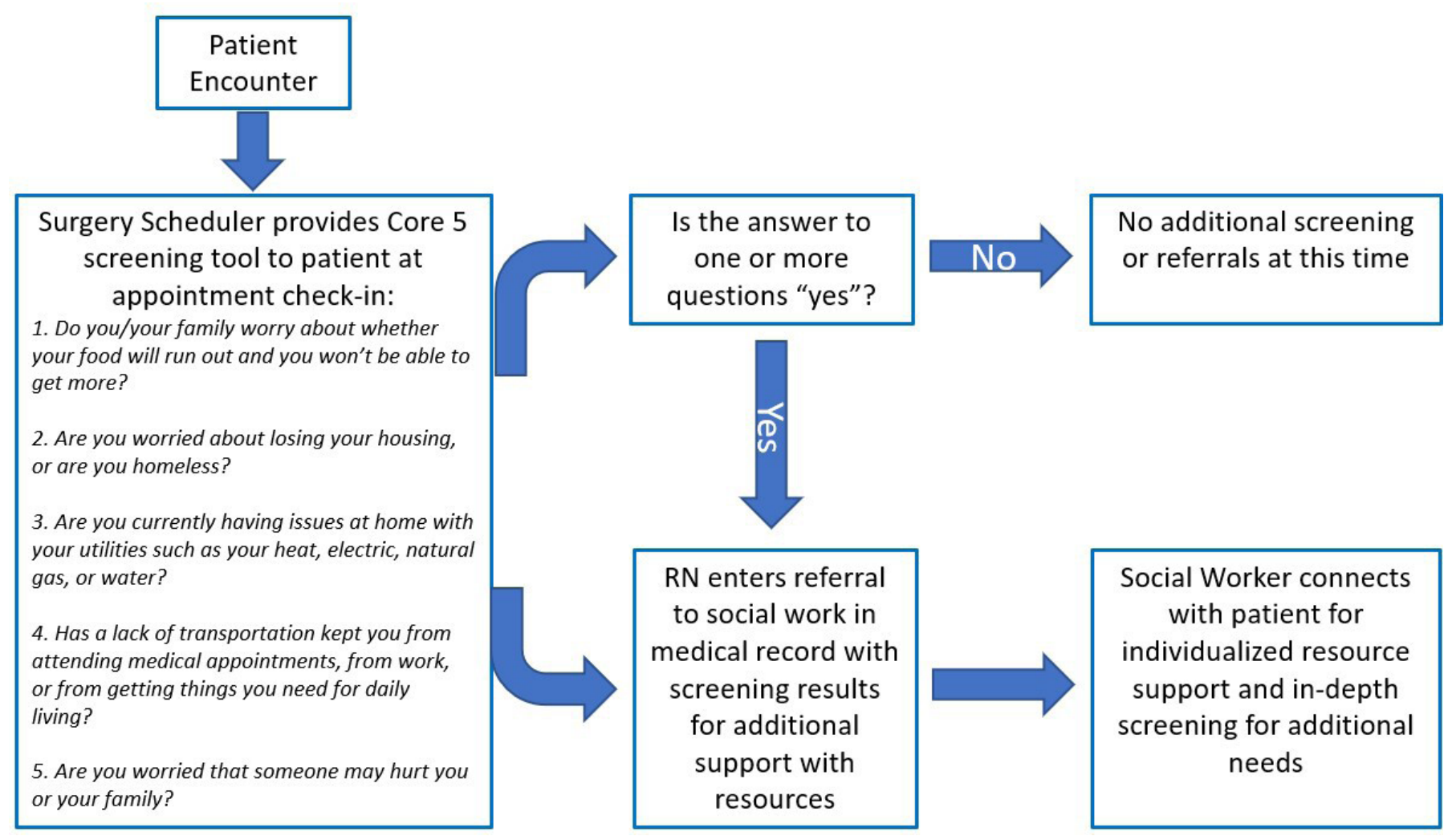

Figure 1 Core 5 social determinants of health screening tool process algorithm for the Surgery Scheduler and Registered Nurse (RN). 
how to introduce the form to patients. If a patient met one of the Core 5 social risk screening criteria questions, a clinic nurse would document the results in the electronic health record (EHR) via a clinical note and would recommend appropriate interventions including a potential referral to a social worker for additional resources. As the screening process was designed to be patient selfcompletion of the screening form, the provider team was not actively involved in the screening process. The project was conducted over a 6 -week period.

\section{PDSA cycle: Study phase}

During the Study phase, analysis of the data was conducted by the PI to determine whether the screening tool accurately identified patients, was usable for staff, and described a beneficial change based on the use of the Core 5 social risk screening tool. Data analysis included patient demographics and social risk factors to describe the population. The feasibility of the Core 5 social risk screening tool was demonstrated based on the successful capture of patient information without patients declining screening, and positive feedback on the staff usability questionnaire. Both of these factors enhanced stakeholder acceptance of the need to consistently screen for social risk factors. The key lesson learnt was that intentional screening of a patient population proved to demonstrate that social risk factors were present and otherwise unaddressed. It was predicted that implementation of the Core 5 social risk screening tool would increase the identification of patients at risk for $\mathrm{SDOH}$. That prediction was met, however, not at the rate anticipated. Next steps will need to focus on increasing use of the Core 5 social risk screening tool and beginning to further determine if there is a link identification of social risk factors, interventions and the ability to reduce outcome metrics such as LOS and readmissions rates.

\section{PDSA cycle: Act phase}

The results and lessons learnt were reported to the organisation's leadership by the PI. The leadership team made the decision to either adopt the use of the Core 5 social risk screening tool, adapt the Core 5 social risk screening tool or abandon the use of the Core 5 social risk screening tool based on project data results. ${ }^{20}$ The leadership recommendation was to spread the tool to all other surgical sections, starting first with orthopaedics and general surgery.

\section{ANALYSES}

Data collection following initiation of use of the Core 5 , allowing time for patient screening, surgery scheduling and hospitalisation after the intake meeting. Data were recorded using an Excel spreadsheet, maintained in a secure drive within the health system's computer system throughout the project period, and archived according to health system standards for securing patient health information.
Descriptive statistics including frequency distributions were calculated to examine six categories of project results. Those categories were: (1) incidence of screening and patient demographics; (2) identified social risk factors; (3) staff evaluation of the Core 5; (4) nursing referrals based on patient responses and (5) sustainability of screening at 1 year.

\section{MEASURES}

\section{Core 5 social risk screening tool}

Qualitative screening results were collected separately for each response on the Core 5 tool and tallied for the total number of social risk factors identified. Patient information that had been entered into their EHR files was compared with the paper copies of the screening tool forms completed by patients to ensure accuracy in transcription and referrals. Review and analysis of screening results were conducted solely by the PI to ensure consistent evaluation standards.

\section{Referral patterns}

Referral patterns were documented based on identified needs and on the time lapsed between when a social need was identified until a referral was placed. Retrospective review of results prevented bias or intervention in the clinical provision of patient care.

Data collection occurred over a 2-month period following initiation of the Core 5 social risk $\mathrm{SDOH}$ screening tool, allowing sufficient time for patient screening, surgery scheduling and hospitalisation. Patient self-report also minimised challenges of staff capacity and training in administering screening. ${ }^{21-23}$ Patient information entered into the EHR was compared with the screening tool forms completed by the patients to ensure accuracy in transcription and referrals. Review and analysis of screening results were conducted solely by the project lead to ensure standard evaluation and eliminate inter-rater variability. Patient input was not involved in the design, implementation or dissemination of this PDSA, however, this project provides the foundation for SDOH work and future community partnership related to social determinants.

\section{Staff feasibility survey}

A staff survey evaluated usability of the tool and perceived benefit of patient screening for SDOH (see online supplemental appendix B). These questions replicated the Columbus Ohio Public Health Department usability measure conducted for the initial evaluation of the tool. ${ }^{18}$

\section{RESULTS}

\section{Incidence of screening and patient demographics}

Fifty-two of the 88 eligible patients were screened (59\%) (table 1). Twenty-four of the patients were women $(46 \%)$. The age range was 22-86 years of age; 27 patients were under 65 years of age. The majority of patients $(n=49$ $(94 \%))$ were from Washington state with 24 residing in 
Table 1. Demographics of Spine Patient Population Participating In Social Determinants of Health Screening.

\begin{tabular}{ll}
\hline $\begin{array}{l}\text { Demographics of spine patient } \\
\text { population (N=52) }\end{array}$ & $\begin{array}{l}\text { Population } \\
\text { description \% (n) }\end{array}$ \\
\hline Female & $46(24)$ \\
Male & $54(28)$ \\
\hline Age & $52(27)$ \\
$\quad 65$ years or less & $48(25)$ \\
$\quad$ Over 65 years & \\
Location & $46(24)$ \\
\hline King county (greater Seattle area) & $94(49)$ \\
$\quad$ Washington state & $6(3)$ \\
\hline $\begin{array}{l}\text { Outside of Washington state } \\
\text { Insurance provider }\end{array}$ & $30(16)$ \\
\hline $\begin{array}{l}\text { Private } \\
\text { Government }\end{array}$ & $64(34)$ \\
\hline Self-pay/no insurance & $6(3)$ \\
\hline Procedure & $\%(a v e r a g e ~ L O S)$ \\
\hline $\begin{array}{l}\text { Major cervicothoracic fusion }(>5 \\
\text { levels) }\end{array}$ & $4(3.5$ days; $\mathrm{n}=2)$ \\
\hline Cervical w/o fusion & $15(1.9$ days; $\mathrm{n}=8)$ \\
\hline Cervical w/fusion & $6(1.2$ days; $\mathrm{n}=3)$ \\
\hline Major lumbar fusion (>5 levels) & $4(4.5$ days; $\mathrm{n}=2)$ \\
\hline
\end{tabular}

LOS, length of stay.

the greater Seattle area and 28 from other areas in the state. Three other participants were from out-of-state. Thirty per cent of patients indicated private insurance providers $(\mathrm{n}=16)$ and compensation, or federal employment benefits. Three patients (6\%) elected to pay out of pocket or did not have insurance benefits.

Six types of procedures were reported based on complexity and vertebrae involvement: (1) major cervicothoracic fusion (more than five levels); (2) cervical surgery without fusion (eg, laminectomy, discectomy, debridement); (3) cervical surgery with fusion; (4) major lumbar fusion (>five levels); (5) lumbar surgery without fusion and (6) lumbar surgery with fusion. Patient procedures were primarily lumbar with $69 \%$ of patients $(n=36)$ screened having a lumbar procedure. Hospital lengths of stay for all procedures ranged from 1.2 to 4.5 days. Sixty-four per cent $(n=34)$ had government insurance including Medicare, dual-eligible, workers' compensation or federal employment benefits. Three patients $(6 \%)$ elected to pay out of pocket or did not have insurance benefits.

\section{Identified social risk factors}

Five of the 52 patients screened (10\%) noted social risk factors. The five varied from the characteristics of the total group with all five being men, having public insurance (Medicare and worker's compensation through the state Labor and Industries department) and residing in the greater Seattle area. Impacts of public insurance were visible in numerous denials for services and a lack of discharge placement options for patients with this type of coverage. One patient with a social risk factor also did not receive surgery during the project timeframe because they had not met physiological criteria for surgery. Hospital stays for each of the four cases identified as having risk factors for $\mathrm{SDOH}$ exceeded the average LOS (ie, 1.4 days for lumbar surgery without fusion, 2.6 days for lumbar surgery with fusion) because of slower progression towards recovery and difficulties with postdischarge planning.

Among the five patients with social risk factors, two reported issues with transportation, one indicated housing needs and one patient also reported risk for self-harm in response to a question regarding concern for safety. Although this was not an expected response to this question, as the question was designed to capture community or domestic violence, the screening identified a critical need related to individual patient safety that otherwise would have been missed.

Two other patients in the sample of $52(3.8 \%)$ required extensive social work intervention who were not identified with the screening process for social risk factors. Both were noted to have baseline physical deconditioning as a result of chronic health issues (ie, stroke and chronic infection), necessitating assistance with activities of daily living that would create challenges for recovery and for postdischarge placement. Of note, one was a non-English speaker, potentially complicating her postsurgical education and postdischarge planning, who was in need of substantial help with her physical recovery.

Four of the five patients who indicated social risks required nursing referrals based on their screening and progression to surgery; the fifth patient did not because he was not eligible for surgery at that time. Nurse referrals to social work services were made for the four going on to surgery. However, social work interventions were provided for only three of the patients. In the fourth case, the referring nurse followed up by linking the patient to appropriate services, ensuring that the patient's needs were met. In all cases, interventions were initiated prior to hospitalisation and/or within 12 hours of arrival at the hospital.

In order to examine the sensitivity of the screening tool, all patients with hospital stays exceeding the average number of days were reviewed for social risk factors that may have been missed. As noted earlier, two more patients were identified with social risk factors. These two patients were hospitalised for 5 and 7 days, respectively, longer than the average LOS for their procedures of 4.5 and 2.1 days for the group as a whole.

\section{Staff usability of the Core 5 screening tool}

Overall, the results of the staff usability survey of the Core 5 screening tool were positive with an average score of 4.4 out of a possible score of 5.0 (figure 2). The highest scores of 5.0 were in response to the following four items 


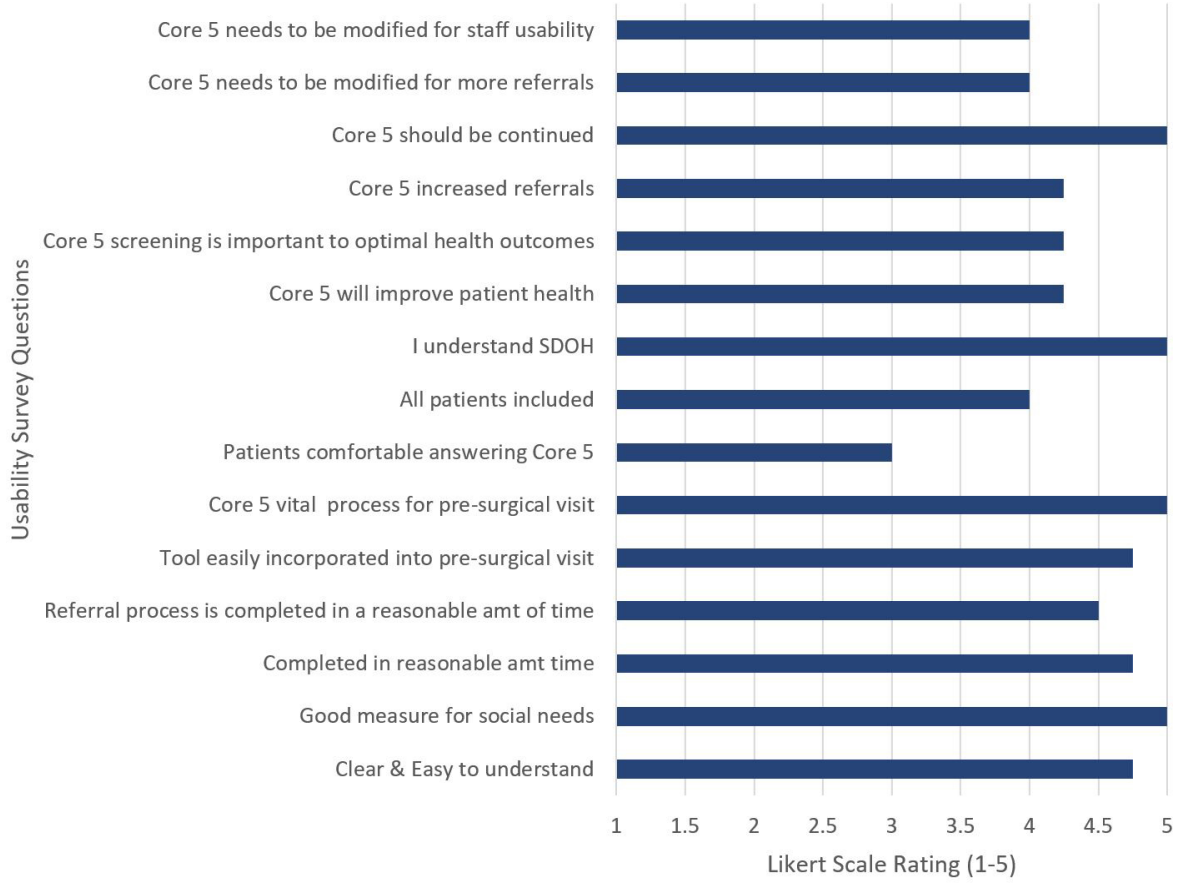

Figure 2 Staff rating of Core 5 tool usability. SDOH, social determinants of health.

of the screening tool: 'The Core 5 should be continued'; 'I understand social determinants of health'; 'The Core 5 tool is a vital process for pre-surgical visits' and 'The Core 5 is a good measure for social needs'. Scores of 3.0 was in response to: 'Patients are comfortable answering the Core 5 questions'.

\section{DISCUSSION}

In this project, we implemented the use of a brief social risk screen, the Core 5, to patients registering for spine surgery in our tertiary spine unit. Our aim was to administer the instrument to $90 \%$ of the patients registering for surgery during a 6 -week trial period. Out of the 88 patients who could have been screened, $59(67 \%)$ completed the Core 5 , a noteworthy improvement from zero.

The results of this pilot project point to several positive outcomes. The Core 5 was easy to include in the intake process and flagged five cases of social risk factors out of the 59 screened, leading to social work referrals for three of the four individuals who were able to progress to surgery. Because of the diligence of a nurse who followed up, the fourth person also was linked with social support.

The staff who evaluated the utility of the Core 5 were very positive about the usability of the tool and about the value of assessing social risks prior to surgery to improve surgical outcomes. Recommendations from participating staff included modifying the tool to ask about the availability of a daily caregiver following surgery. This suggestion would address additional social support needs seen in the two patients who, though not indicating risk factors on the original Core 5, had noteworthy needs for follow-up support that had contributed to their extended hospital stays.
Further examination of the intake process for the patients who were not screened showed variability between surgery schedulers that may indicate that some had insufficient familiarity with the screening tool or discomfort regarding the nature of the questions. Although biweekly discussion with the teams to check on implementation progress during the project did not reveal challenges in screening or patient resistance, more work is needed to understand the barriers to full implementation.

The finding that this pilot project was able to quantify patient social risk factors within this narrow population illustrated the need for broader organisational work to address social determinants. Additional presurgical clinics in the hospital are now screening for social risk factors and all in-patients are completing the Core 5 . Likewise, EHR resources have been allocated in support of this work including establishing documentation fields for social risk factors (vs a clinical note), placing social determinant risk factors on the demographic banner in the EHR and colocating SDOH risk factors with a variety of other demographic, language and other data to improve provision of individualised, holistic patient care. The positive response to this tool validates the key criteria used in selecting a usable tool: abbreviated length and targeted SDOH domains.

Future steps to increase social risk screening will be to add two additional questions to the Core 5 screening tool regarding social support following hospitalisation and financial concerns specifically related to medications. The current Core 5 screening questions will remain with the aim to capture risks of social isolation and additional connections between finances and treatment. Future work is needed on ways to further streamline workflow 
procedures in order to increase usability of social risk screening and to have screening information visible across clinic and hospital settings to optimise the application of steps to provide necessary support.

In prior research, screening for social risk has been shown to have beneficial impact to patient experiences and potentially to patient hospital outcomes. ${ }^{13} 15$ 24-29

Limitations of this project include: (1) a small sample size, (2) failure to meet the project goal of $90 \%$ implementation, (3) small scope of the screening PDSA, (4) visibility of screening information and (5) quality improvement projects are not designed to contribute to generalisable knowledge as are clinical research studies which challenge the reliability and validity of such initiatives. ${ }^{30}$ Further work will be needed to increase the visibility of self-report patient responses within the medical record for all teams to clearly see screening results and improve interventions with patients, work that was not possible in one PDSA cycle.

\section{CONCLUSION}

In this project, the Core 5 social risk screening tool was shown to identify patients currently experiencing social risk factors and to be usable by staff within a hospital clinic workflow. Further, the staff participating reported high value of the screening process and tool itself, mirroring the sense of urgency underscored in the research on the role of social determinants of health on patient outcomes and resource utilisation. Future work should continue to focus on testing the use of this tool in other ambulatory and tertiary settings to further investigate the value of collecting social risk information for the purpose of intervention and improving health outcomes.

Acknowledgements Many thanks to SLL and RW for their support for this project and continuing input. Additionally, thank you to Rebecca Pizzitola for your introduction to the Core 5 Tool and incorporation of this SDOH screening project in the work of the Washington Center for Nursing.

Contributors $A B$ conceived and designed the study, analysed and interpreted the data. AB, SL-L, RW and CB critically revised the manuscript for important intellectual content. All authors approved the final version of the manuscript.

Funding The authors have not declared a specific grant for this research from any funding agency in the public, commercial or not-for-profit sectors.

Competing interests None declared.

Patient consent for publication Not required.

Ethics approval The VMMC institutional review board granted this project an exemption from ethics review.

Provenance and peer review Not commissioned; externally peer reviewed.

Supplemental material This content has been supplied by the author(s). It has not been vetted by BMJ Publishing Group Limited (BMJ) and may not have been peer-reviewed. Any opinions or recommendations discussed are solely those of the author(s) and are not endorsed by BMJ. BMJ disclaims all liability and responsibility arising from any reliance placed on the content. Where the content includes any translated material, BMJ does not warrant the accuracy and reliability of the translations (including but not limited to local regulations, clinical guidelines, terminology, drug names and drug dosages), and is not responsible for any error and/or omissions arising from translation and adaptation or otherwise.

Open access This is an open access article distributed in accordance with the Creative Commons Attribution Non Commercial (CC BY-NC 4.0) license, which permits others to distribute, remix, adapt, build upon this work non-commercially, and license their derivative works on different terms, provided the original work is properly cited, appropriate credit is given, any changes made indicated, and the use is non-commercial. See: http://creativecommons.org/licenses/by-nc/4.0/.

\section{REFERENCES}

1 Andermann A, CLEAR Collaboration. Taking action on the social determinants of health in clinical practice: a framework for health professionals. CMAJ 2016;188:E474-83.

2 Gottlieb LM, Wing H, Adler NE. A systematic review of interventions on patients' social and economic needs. Am J Prev Med 2017;53:719-29.

3 Ohanian A, McConnell D, AJMC. The ROI of addressing social determinants of health, 2018. Available: https://www.ajmc.com/ contributor/ara-ohanian/2018/01/the-roi-of-addressing-socialdeterminants-of-health [Accessed 08 Dec 2020].

4 Virginia Mason Health System. Length of stay internal analytics report, 2019

5 Washington state health assessment, 2018. Washington state department of health. Available: https://www.doh.wa.gov/Portals/1/ Documents/Pubs/78945-SHA.pdf

6 Bree Collaborative. Lumbar fusion surgical bundle. rev ED, 2014. Available: Lumbar-Fusion-Bundle-Final-14-09.pdf (breecollaborative. org)

7 World Health Organization. About social determinants of health. Available: https://www.who.int/social_determinants/en/

8 Lofters AK, Schuler A, Slater M, et al. Using self-reported data on the social determinants of health in primary care to identify cancer screening disparities: opportunities and challenges. BMC Fam Pract 2017;18:2-11.

9 Artiga S, Hinton E. Beyond health care: the role of social determinants in promoting health and health equity, 10 May 2018. Henry J Kaiser family Foundation. Available: https://www.kff.org/ disparities-policy/issue-brief/beyond-health-care-the-role-of-socialdeterminants-in-promoting-health-and-health-equity/ [Accessed 07 Dec 2020].

10 Buja A, Canavese D, Furlan P, et al. Are Hospital process quality indicators influenced by socio-demographic health determinants. Eur J Public Health 2015;25:759-65.

11 Kent J. Social determinants of health impact severity of chronic disease, Feb 2019. Population health news. Available: https:// healthitanalytics.com/news/social-determinants-of-health-impactseverity-of-chronic-disease [Accessed 07 Dec 2020].

12 Marmot M, Bell R. Social inequalities in health: a proper concern of epidemiology. Ann Epidemiol 2016;26:238-40.

13 Meddings J, Reichert H, Smith SN, et al. The impact of disability and social determinants of health on condition-specific readmissions beyond Medicare risk adjustments: a cohort study. J Gen Intern Med 2017;32:71-80.

14 Hero JO, Zaslavsky AM, Blendon RJ. The United States leads other nations in differences by income in perceptions of health and health care. Health Aff 2017;36:1032-40.

15 Hatef E, Searle KM, Predmore Z, et al. The impact of social determinants of health on hospitalization in the Veterans health administration. Am J Prev Med 2019;56:811-8.

16 Centers for Medicare and Medicaid. The accountable health communities health-related social needs screening tool, 2019. Available: https://innovation.cms.gov/files/worksheets/ahcmscreeningtool.pdf

17 Prevention Institute. Thrive (tool for health and resilience in vulnerable environments). Available: https://www.preventioninstitute.org/tools/ thrive-tool-health-resilience-vulnerable-environments

18 Ohio Action Coalition. "Core determinants of health screening tool, aka the "Core 5". Available: https://cdn.ymaws.com/www. ohioleaguefornursing.org/resource/resmgr/ohio_action_coalition/ PH_Nurse_Leader_Project/Attachment_B_CDH_Screening_T.pdf [Accessed 15 Dec 2020].

19 Associates for process improvement, 2020. Available: https://www. apiweb.org/ [Accessed 08 Dec 2020].

20 Leming-Lee Treasa 'Susie', Watters R. Translation of evidence-based practice: quality improvement and patient safety. Nurs Clin North Am 2019;54:1-20.

21 National Association of Community Health Centers. PRAPARE (Protocol for responding to and assessing patients' assets, risks, and experiences), 2020. Available: https://www.nachc.org/research-anddata/prapare

22 Gottlieb L, Hessler D, Long D, et al. A randomized trial on screening for social determinants of health: the iScreen study. Pediatrics 2014;134:e1611-8. 
23 Virapongse A, Misky GJ. Self-identified social determinants of health during transitions of care in the medically underserved: a narrative review. J Gen Intern Med 2018;33:1959-67.

24 Chen S, White R, Tangel V, et al. Sociodemographic characteristics predict readmission rates after lumbar spinal fusion surgery. Pain Med 2019;0:1-14.

25 Safaee MM, Scheer JK, Ailon T, et al. Predictive modeling of length of hospital stay following adult spinal deformity correction: Analysis of 653 patients with an accuracy of $75 \%$ within 2 days. World Neurosurg 2018;115:e422-7.

26 Hewner S, Casucci S, Castner J. The roles of chronic disease complexity, health system integration, and care management in postdischarge healthcare utilization in a low-income population. Res Nurs Health 2016;39:215-28.
27 Navathe AS, Zhong F, Lei VJ, et al. Hospital readmission and social risk factors identified from physician notes. Health Serv Res 2018;53:1110-36.

28 Hewner S, Sullivan SS, Yu G. Reducing emergency room visits and in-hospitalizations by implementing best practice for transitional care using innovative technology and big data. Worldviews Evid Based Nurs 2018;15:170-7.

29 Joynt Maddox KE, Reidhead M, Hu J, et al. Adjusting for social risk factors impacts performance and penalties in the hospital readmissions reduction program. Health Serv Res 2019;54:327-36.

30 Casartett D, Karlaswish J, Sugarman J. Determining when quality improvement initiatives should be considered research: proposed criteria and potential implications. JAMA 2009;283:2275-80. 